\title{
Incidental Finding of Mediastinal Mass in A Patient with A Confirmed Diagnosis of Moderate to Severe COVID-19: Case Report
}

\author{
Julián Rondón-Carvajal ${ }^{1,2 *}$ | Federico Rodríguez-Vega ${ }^{1,2}$ | Lucy Pérez-Lugo ${ }^{1,2}$ | Karina Madera- \\ Gutiérrez $^{1,2}$ | Fair Clavijo-Tinoco ${ }^{1,2}$
}

*Correspondence: Julián Rondón-Carvajal

Address: ${ }^{1}$ Intermediate respiratory care unit. SURA EPS. CES clinic. Medellín, Colombia; 2Specialist in Internal Medicine, IPS

SURA Health Services. Medellín, Colombia

e-mail $\bowtie$ : jrondon@sura.com.co

ORCID-ID: https://orcid.org/0000-0001-9804-8990

Received: 26 August 2020; Accepted: 31 August 2020

Copyright: (C) 2020 Rondón-Carvajal J. This is an open-access article distributed under the terms of the Creative Commons Attribution License, which permits unrestricted use, distribution, and reproduction in any medium, provided that the original work is properly cited.

\section{ABSTRACT}

Positive airway pressure ventilation systems are a therapeutic option in patients with confirmed SARS-CoV2 infection, who meet both clinical and biochemical criteria to be classified as moderate to severe cases, in order to avoid and / or delay progression to acute ventilatory failure. However, its success largely depends on a correct selection of the clinical setting. We present the case of a patient taken to non-invasive mechanical ventilation with slow positive end-expiratory pressure (PEEP) titration indicated in the event of persistent tachypnea despite correcting hypoxemia with conventional oxygen therapy, subsequently documenting a large mass in the anterior mediastinum, unknown to date. The patient had a favorable evolution from the clinical point of view. At the end, the eligibility criteria for considering the use of noninvasive mechanical ventilation in patients with COVID-19 will be discussed in a concise manner.

Keywords: COVID-19, SARS-CoV-2, Mediastinal Diseases, Noninvasive Positive Pressure Ventilation

\section{Introduction}

The COVID-19 pandemic has become a huge challenge for the different health systems in the world and Colombia has not been the exception. As of August 20, 513,719 confirmed cases of the disease and about 16,000 deaths were registered in the country (Ministerio de Salud y Protección Social, República de Colombia, 2020), which places us among the most affected countries in Latin America. The prevention and management are very important issues to control COVID-19. Therefore, there is a great need for the collective efforts of the public and the government. The regular and the proper care of the homes and hospitals are very important to control this disaster (Ali I and Alharbi 0, 2020). However, the other side of the pandemic has to do with chronic non-transmittable diseases, including neoplasms both solid tumors and hematolymphoids, which require opportunity in diagnosis and treatment to have a positive impact in prognostic terms (Ali I and Alharbi O, 2020; Sternberg et al., 2020). 
On the other hand, oxygen therapy is a fundamental part of supportive treatment for patients with COVID-19 and acute hypoxemic ventilatory failure (Zhang et al., 2020; Tinelli et al., 2019). The different clinical practice guidelines recommend titrating the oxygen flow according to the severity of the hypoxia, suggesting starting conventional supplemental oxygen if the peripheral oxygen saturation $\left(\mathrm{SpO}_{2}\right)$ is less than $90 \%$ and adjusting it to maintain $\mathrm{SpO}_{2}$ greater than $94 \%$ until reaching high flows (greater than 15 liters per minute) and later, consider other non-invasive supports that allow delaying and even avoiding invasive mechanical ventilation (Zhang et al., 2020), among which are the high-flow nasal cannula and non-invasive mechanical ventilation (NIMV) with its dynamic interfaces: oronasal masks, face masks or Helmet-type mask as well as a device to administer positive pressure. The choice of one over the other will depend on the clinical profile of the particular patient (Tinelli et al., 2019).

\section{Methods}

A detailed review of the clinical history was carried out, including gas variables and the images taken on the patient during their stay in the intermediate respiratory care unit. A literature search was conducted for cases similar to ours. It was decided to defer histopathological study of the mass, once it was transferred to the hospitalization room.

\section{Case Report}

A 68-year-old man with a 19-year history of colorectal cancer, stage I, requiring localized surgical resection in sigmoid colon with termino-terminal anastomosis, without complementary chemotherapy or radiotherapy. Loss of follow-up by oncology service to date. Additionally, arterial hypertension and dyslipidemia, in management with losartan/hydrochlorothiazide 50/12.5 mg BID and atorvastatin 40 mg QD. He consulted to the emergency department for 7 days of non-productive or hemoptoic cough, associated with piercing-type chest pain and emetic episodes without hematemesis and fainting. In view of this clinical picture, he was asked for an electrocardiogram (EKG) and troponin i (TpI), ruling out symptoms of cardiogenic origin; furthermore, a simple cranial CT scan without relevant findings. Considering the current epidemiological context, an antigenic test for SARS-CoV2 was requested on day 7, which was positive.

On admission, showed marked tachypnea, with respiratory rate: 35-40 rpm, blood pressure 137/74 mmHg, heart rate: $115 \mathrm{bpm}, \mathrm{SaO}_{2}$ 91\% with $\mathrm{FiO} 36 \%$, without use of accessory muscles, requiring supplementary oxygen by simple mask at $5 \mathrm{~L} / \mathrm{min}$. Arterial gases compatible with type I respiratory failure, without acid-base imbalance, with a ratio $\mathrm{PaO}_{2} / \mathrm{FiO}_{2}: 201$, in relation to moderate oxygenation disorder (ARDS Definition Task Force, 2012). 
Chest radiography with mixed opacities of alveolar predominance with extensive left parahiliary involvement, meeting operational definition criteria for severe COVID-19 disease, Hence was decided to transfer to an intermediate respiratory care unit (IRCU).

Blood examination showed hemoleucogram with leukocytosis, neutrophilia, moderate lymphopenia, without thrombocytopenia (Table 1). Prognostic markers were for COVID-19 (Velavan et al., 2020): lactate dehydrogenase, D-dimer and ferritin were elevated.

Table 1: Laboratory data

\begin{tabular}{|c|c|c|}
\hline Variable & Values & Reference range \\
\hline Leukocytes $\left(10^{3} / \mathrm{mL}\right)$ & 16930 & $4400-11000$ \\
\hline Neutrophils $\left(1^{3} / \mathrm{mL}\right)$ & 15190 & $2000-7500$ \\
\hline Lymphocytes $\left(10^{3} / \mathrm{mL}\right)$ & 919 & $1000-4000$ \\
\hline Platelets $\left(10^{3} / \mathrm{mL}\right)$ & 246000 & $150000-450000$ \\
\hline Hemoglobin (g/dL) & 13.8 & $13-16$ \\
\hline C reactive protein $(\mathrm{mg} / \mathrm{L})$ & 37.85 & $<6$ \\
\hline D dimer $(\mathrm{ng} / \mathrm{mL})$ & 1100 & $<500$ \\
\hline Ferritin (mcg/L) & 501 & $30-500$ \\
\hline Lactate dehydrogenase (U/L) & 325.5 & $88-230$ \\
\hline Creatinine (mg/dL) & 1.27 & $0.6-1.2$ \\
\hline Blood urea nitrogen (mg/dL) & 20.4 & $8-20$ \\
\hline Total bilirubin (mg/dL) & 0.78 & $0.1-1-2$ \\
\hline Glucose (mg/dL) & 117 & $60-110$ \\
\hline $\operatorname{ALT}(\mathrm{U} / \mathrm{L})$ & 14.8 & $0-35$ \\
\hline $\operatorname{AST}(U / L)$ & 18.9 & $0-35$ \\
\hline $\mathrm{PO}_{2}$ (mmHg) & 67.5 & $80-100$ \\
\hline $\mathrm{PCO}_{2}$ (mmHg) & 37 & $35-45$ \\
\hline $\mathrm{HCO}_{3}(\mathrm{meq} / \mathrm{L})$ & 23.1 & $22-26$ \\
\hline
\end{tabular}

Table 2: Non-invasive mechanical ventilation (NPPV) parameters

\begin{tabular}{|c|c|c|c|c|c|c|c|}
\hline NPPV & PS (cmH20) & PEEP (cmH20) & RR (rpm) & SPO2 (\%) & VT (mL/Kg) & FIO2 (\%) & Device \\
\hline $\begin{array}{l}\text { First Cycle } \\
\text { CPAP }\end{array}$ & 12 & 6 & 0 & 90 & 6 & 100 & Oronasal mask \\
\hline $\begin{array}{l}\text { Second Cycle } \\
\text { CPAP }\end{array}$ & 14 & 10 & 0 & 95 & 7 & 70 & Oronasal mask \\
\hline
\end{tabular}

Table 3: High-flow nasal cannula (HFNC) parameters

\begin{tabular}{|l|c|c|c|c|}
\hline \multicolumn{1}{|c|}{ HFNC } & Flow (L/min) & Temperature $\left({ }^{\circ}\right.$ F) & FIO2 (\%) & SP02 (\%) \\
\hline Adaptation Phase & 60 & 98.6 & 100 & 94 \\
\hline Maintenance Phase & 50 & 98.6 & 60 & 97 \\
\hline
\end{tabular}


Patient persisted clinically impaired, with severe oxygenation disorder, that is, with a ratio $\mathrm{PaO}_{2} / \mathrm{FiO}_{2}$ : $91 \mathrm{mmHg}$, which required oxygen supplementation by reservoir mask at $15 \mathrm{lt} / \mathrm{min}$ and antimicrobial coverage for bacterial coninfection common germs with ampicillin/sulbactam IV was administered considering clinical data such as fever, unimprovement on clinical behavior, as well as laboratory data like leukocytosis, neutrophilia and elevated C-reactive protein. After 2 hours with persistent tachypnea, respiratory support with non-invasive mechanical ventilation was initiated using Helmet as an interface with conservative parameters as described on Table 2, with $4 \mathrm{X} 4$ cycles interspersed with a reservoir mask at $10 \mathrm{~L} / \mathrm{min}$, it was adjusted an increased PEEP and $\mathrm{FiO}_{2}$ reduction as showed at Table 2. The patient presented oxygenation parameters improvement with a ratio $\mathrm{PaO}_{2} / \mathrm{FiO}_{2}$ : $112 \mathrm{mmHg}$, respiratory rate: 20-23 rpm, without use of accessory muscles and oxygen saturation $\left(\mathrm{SaO}_{2}\right)$ between $95-97 \%$.

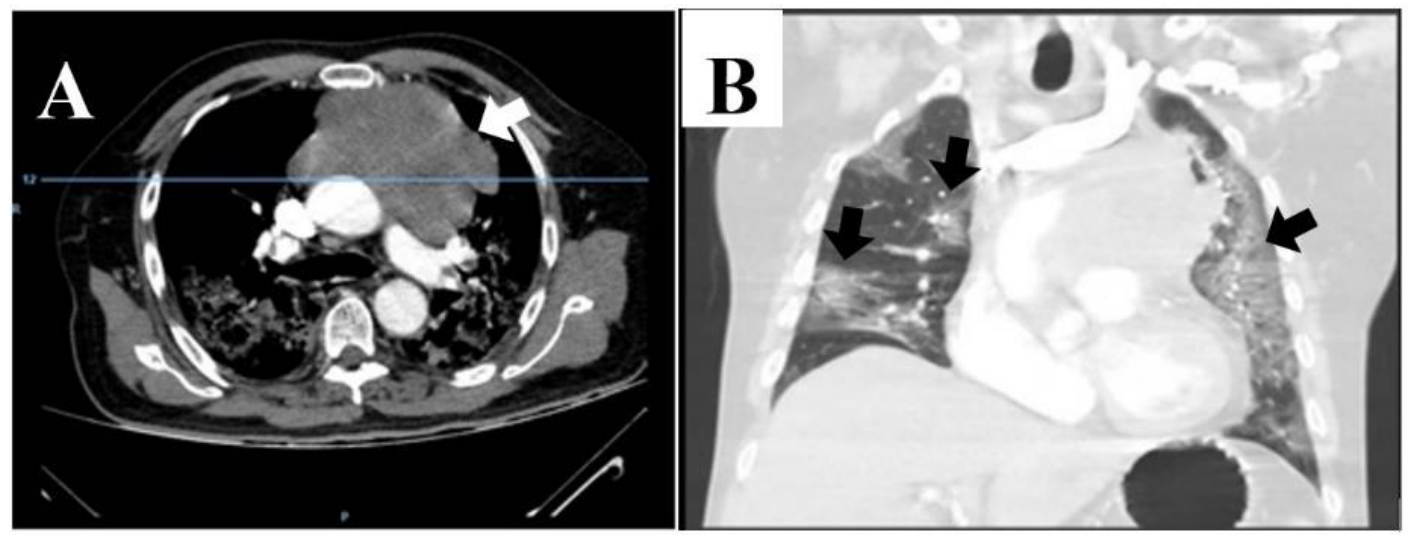

Figure 1: Angiotomography: A. Solid mass in the anterior mediastinum, homogeneous, 110x80 mm, without calcifications or fat components, compressing and displacing large vessels in the posterior direction, without infiltration of the same (white arrow). B. Extensive patchy areas of frosted glass greater than $3 \mathrm{~cm}$, confluent, accompanied by extensive "cobblestone" pattern (black arrow)

In order to evaluate other underlying causes of hypoxemia (Sarkar et al., 2017), angiotomography (angioTAC) was performed, which ruled out acute pulmonary embolism, documenting extensive patchy areas of frosted glass larger than $3 \mathrm{~cm}$, confluent, accompanied by extensive "cobblestone" pattern and bilateral basal consolidations, as well as solid mass in anterior mediastinum, homogeneous, 110x80 $\mathrm{mm}$, without calcifications or fat component, compressing and displacing large vessels in posterior direction, without infiltration (Fig. 1).

In view of the presence of the described radiological findings and physical examination with bibasal hypophonesis, crepitus in both pulmonary fields, without neck edema, without jugular ingurgitation at 45 degrees, palpable goiter or testicular hypotrophy. Considering the presence of mass in anterior mediastinum in a patient with non-invasive mechanical ventilation, it was decided to start 
assistance with high flow nasal cannula (Tinelli et al., 2019; Helviz and Einav, 2018), in order to reduce dead space, improve diaphragmatic work, $\mathrm{CO}_{2}$ wash out and avoid mechanical complications by offering low PEEP, initial parameters were configurated as discribed on Table 3 during 6 hours, under $\mathrm{FiO}_{2}$ titration according to $\mathrm{SPO}_{2}$. After 6 hours, a remarkable improvement on the respiratory dynamics was achieved, obtaining FR 25 rpm, $\mathrm{SPO}_{2}$ 96\% without accessory muscles use. The maintenance phase was completed, without achieving the release of the high flow system until 5 days later, with stable evolution until COVID-19 resolution.

\section{Discussion}

To date, there are no case reports that relate the debut of an anterior mediastinal mass in the same patient with a confirmed molecular diagnosis of COVID-19. There were also no reports of experience between ventilation with positive airway pressure in patients with lesions of these characteristics, so this case report is anecdotal, while allowing us to specify selection criteria for the use of this therapy. Only one suspected case of COVID-19 was documented in a 32-year-old man with a large mediastinal mass after being tested on 3 occasions for SARS-CoV2 infection, on suspicion of COVID-19, with a final histopathological diagnosis compatible with a germ cell tumor (O'Brien and Power, 2020) and the case of a patient with a known diagnosis of primary mediastinal diffuse B-cell lymphoma, who developed COVID after intensive chemotherapy with DA-EPOCH-R protocol (etoposide, prednisone, vincristine, cyclophosphamide, doxorubicin, and adjusted-dose rituximab) (Li et al., 2020).

Although in patients with hypoxemic ventilatory failure secondary to COVID-19 infection and under strict monitoring, the high-flow nasal cannula (CNAF) is recommended over non-invasive mechanical ventilation (NIMV), since CNAF seems to be associated with a lower risk of intubation, nosocomial infection and greater comfort for the patients, the use of NIMV (with interface helmet) compared to CNAF, seems to have an improvement in the parameters of the $\mathrm{PaO}_{2} / \mathrm{FIO}_{2}$ ratio (PAFI), inspiratory effort and dyspnea (Grieco et al., 2020x1), as in this case, where after two 4-hour cycles with titratable ventilator parameters, an improvement in ventilatory mechanics was achieved, but given the persistence of a severe oxygenation disorder due to a persistent fall in PAFI, an angiotomography to evaluate other causes of persistent hypoxemia, which ruled out pulmonary embolism, having as an incidental finding of mediastinal mass not observed in portable chest X-ray, indicating suspension of NIMV.

Positive airway pressure has the benefits of reducing airway resistance, increasing the final volumes of pulmonary inspiration, as well as favoring alveolar recruitment, presenting as risks the possibility of barotrauma, so that in hypoxemic ventilatory failure it is not it is not recommended to use 
PEEP greater than $10 \mathrm{mmHg}$, unless it is required to adjust other ventilatory parameters due to the patient's condition, and to maintain low tidal volume levels (Alhazzani et al., 2020; Bhimraj et al., 2020; Miller et al., 2020; Frat et al., 2017).

The potential risk of transmission of the infection must also be considered, so to carry out any of the aforementioned interventions, a safe environment must be available to avoid contagion from other patients and / or health personnel, in an area that has with adequate ventilation or with negative pressure mechanisms, without ever dispensing with protection measures against aerosols (Jin et al., 2020; Chawla et al., 2020).

The following are potential clinical scenarios to consider the use of NIMV in patients with respiratory failure secondary to COVID-19 (Chawla et al., 2020; Nasibova and Pashayev, 2020):

- Acute hypercapnic respiratory failure (patients with respiratory muscle fatigue and hypoventilation): $\mathrm{PaCO}_{2}>45 \mathrm{~mm} \mathrm{Hg}$, $\mathrm{pH}<7.3, \mathrm{PaO}_{2} / \mathrm{FiO}_{2}<200$.

- Severe exacerbation of chronic obstructive pulmonary disease (COPD), with severe respiratory acidosis $(\mathrm{pH}<7.30)$

- Acute cardiogenic pulmonary edema

- Hypoxemic respiratory failure: between 15-35\% patients during the first minutes or hours of NIV, no improvement by clinical indicators and gas exchange is observed or the procedure is poorly tolerated (Nasibova and Pashayev, 2020). Usually, a respiratory support session of 2-3 hours is sufficient to predict the success of the NIV or response to the NIV. In normal practice, the effectiveness of NIV therapy is obvious with a simple examination - there is a decrease in the frequency of respiratory movements and the work of auxiliary respiratory muscles. Objective markers of the effectiveness of mask ventilation are changes in arterial blood gas parameters, given by an increase in $\mathrm{pH}$ and a decrease in $\mathrm{PaCO}_{2}$. In our case, the use of a helmet as an interface for NIV administration sought to improve the parameters of the $\mathrm{PaO}_{2} / \mathrm{FIO}_{2}$ ratio (PAFI), inspiratory effort and dyspnea.

Although the evidence to date favors the use of a high-flow nasal cannula over NIMV systems programmed in CPAP mode, it is clear that a one-size-fits-all approach to AHRF is misguided. Choosing the right noninvasive oxygen support likely requires a precision-based approach that matches a given strategy to the observed phenotype of AHRF coupled with incorporating clinician experience and comfort with each technology. For instance, perhaps lung injury that is nonresponsive to PEEP is best served with a trial of HFNC. Alternatively, NIV may be considered if the lung injury seems PEEP responsive, with milder hypoxemia $\left(\mathrm{PaO}_{2} / \mathrm{FIO}_{2}\right.$ ratio $\left.>200\right)$ reserved for the face mask interface and severe hypoxemia with a prolonged need of NIV application reserved for helmet (Patel et al., 2020). 
In our case, the patient presented even $\mathrm{PaO}_{2} / \mathrm{FIO}_{2}$ ratio values between 100 and 150 when NIMV was indicated with slow PEEP titration (6-10 $\mathrm{cmH}_{2} 0$ ), which probably prevented respiratory deterioration in the context of the incidental finding of a mass in the mediastinum, pending to typify.

\section{Conclusions}

- Intermediate respiratory care units (IRCUs) have gained notable relevance during the COVID-19 pandemic as containment spaces for patients with a moderate to severe course of the disease, in whom they seek to delay the use of invasive mechanical ventilation, achieving in many cases even avoid this situation.

- Although current evidence in patients with hypoxemic ventilatory failure secondary to COVID19 disease favors the use of CNAF over NIV under monitoring to identify the failure and the need for IOT, since the CNAF seems to be associated with a lower risk of intubation, nosocomial infection and greater comfort for patients, the use of NIV with Helmet as an interface is an alternative management in these patients with indication of high flow oxygen systems.

- Clinical and gasimetric evaluation in patients with COVID-19 should be dynamic, in order to rule out thrombotic and infectious complications even in the course of the disease. In this process we could find relative contraindications for the use of positive airway pressure, such as a mediastinal mass.

\section{Declaration of Interests and Ethical Approval}

The authors declare that they have no competing financial interests or personal relationships that could have appeared to influence the work reported in this paper. The patient in this manuscript has given informed consent to publication of their case details. The academic coordination office of EPS SURA evaluated and authorized the publication of this manuscript.

\section{References}

Alhazzani W, Møller MH, Arabi YM. Surviving Sepsis Campaign: guidelines on the management of critically ill adults with Coronavirus Disease 2019 (COVID-19). Intensive Care Med 2020; 46: 854-887.

Ali I and Alharbi O. COVID-19: Disease, management, treatment, and social impact. The Science of the total environment 2020; 728: 138861.

ARDS Definition Task Force, Ranieri VM, Rubenfeld GD, Thompson BT, Ferguson ND, Caldwell E. Acute respiratory distress syndrome. JAMA 2012; 307: 2526-2533.

Bhimraj A, Morgan RL, Shumaker AH, Lavergne V, Baden L, Cheng VC, Edwards KM, Gandhi R, Muller WJ, O’Horo JC, Shoham S. Infectious diseases Society of America guidelines on the treatment and management of patients with COVID-19. Clin Infect Dis 2020; pp: 478.

Chawla R, Dixit SB, Zirpe KG, Chaudhry D, Khilnani GC, Mehta Y, Khatib KI, Jagiasi BG, Chanchalani G, Mishra RC, 
Samavedam S. ISCCM guidelines for the use of non-invasive ventilation in acute respiratory failure in adult ICUs. Indian J Crit Care Med 2020; 24: S61-S81.

Frat JP, Coudroy R, Marjanovic N, Thille AW. High-flow nasal oxygen therapy and noninvasive ventilation in the management of acute hypoxemic respiratory failure. Ann Transl Med 2017; 5: 297.

Grieco DL, Menga LS, Raggi V, Bongiovanni F, Anzellotti GM, Tanzarella ES, Bocci MG, Mercurio G, Dell'Anna AM, Eleuteri D, Bello G. Physiological comparison of high-flow nasal cannula and helmet noninvasive ventilation in acute hypoxemic respiratory failure. American journal of respiratory and critical care medicine 2020; 201: 303-312.

Helviz Y, Einav S. A systematic review of the high-flow nasal cannula for adult patients. Critical care (London, England) 2018; 22: 71.

Jin YH, Cai L, Cheng ZS, Cheng H, Deng T, Fan YP, Fang C, Huang D, Huang LQ, Huang Q, Han Y. A rapid advice guideline for the diagnosis and treatment of 2019 novel coronavirus (2019-nCoV) infected pneumonia (standard version). Mil Med Res 2020; 7: 4 .

Li Q, Zhu F, Xiao Y, Liu T, Liu X, Wu G, Zhang L. A Primary Mediastinal Large B-Cell Lymphoma Patient With COVID-19 Infection After Intensive Immunochemotherapy: A Case Report. Frontiers in Oncology 2020; 10: 924.

Miller DC, Bime C, Partharsarathy S, Mosier JM. High-Flow Oxygen Therapy Concepts: Time to Standardize Nomenclature and Avoid Confusion. J Intensive Care Med 2020; 35: 519-523.

Ministerio de Salud y Protección Social, República de Colombia. Situación actual en Colombia: Nuevo Coronavirus (COVID-19), 20 de agosto de 2020. Sistema Integrado de Información de la Protección Social (SISPRO). URL: https://sig.sispro.gov.co/SituacionCovid/

Nasibova EM and Pashayev CN. The Use of Non-Invasive Ventilation (NIV) in the Treatment of Patients with COVID-19.J Intensive \& Crit Care 2020; 6: 5.

O'Brien T, Power DG. Mediastinal germ cell tumour during the COVID-19 pandemic. BMJ Case Reports CP 2020; 13 : e237003.

Patel BK, Kress JP, Hall JB. Alternatives to Invasive Ventilation in the COVID-19 Pandemic. JAMA 2020; 324: 43-44.

Sarkar M, Niranjan N, Banyal PK. Mechanisms of hypoxemia. Lung India: official organ of Indian Chest Society 2017; 34: 47-60.

Sternberg C, Andrade TL, Nova AP, Fiscina BV, Fernandes AP, Alves CD, Alves AB, Hizumi LO, Dias SS, Nobre P, Calabrich A. Oncology practice during COVID-19 pandemic: a fast response is the best response. Rev Assoc Med Bras 2020; 66(3):338-344.

Tinelli V, Cabrini L, Fominskiy E, Franchini S, Ferrante L, Ball L, Pelosi P, Landoni G, Zangrillo A, Secchi A. High flow nasal cannula oxygen vs. conventional oxygen therapy and noninvasive ventilation in emergency department patients: a systematic review and meta-analysis. J Emerg Med 2019; 57: 322-328.

Velavan TP, Meyer CG. Mild versus severe COVID-19: laboratory markers. International Journal of Infectious Diseases 2020; 95: 304-307.

Zhang G, Hu C, Luo L, Fang F, Chen Y, Li J, Peng Z, Pan H. Clinical features and short-term outcomes of 221 patients with COVID-19 in Wuhan, China. J Clin Virol 2020; 127: 104364. 